\title{
Prediction of unconventional magnetism in doped $\mathrm{FeSb}_{2}$
}

\author{
I. I. Mazin, ${ }^{1,2}$ K. Koepernik,${ }^{3}$ M.D. Johannes, ${ }^{4}$ Rafael González-Hernández, ${ }^{5,6}$ and Libor Šmejkal ${ }^{6,7}$ \\ ${ }^{1}$ Department of Physics and Astronomy, George Mason University, Fairfax, USA \\ ${ }^{2}$ Center for Quantum Science and Engineering, George Mason University, Fairfax, USA \\ ${ }^{3}$ Institute for Theoretical Solid State Physics, IFW Dresden, 01069 Dresden, Germany \\ ${ }^{4}$ Center for Computational Materials Science, Naval Research Laboratory, Washington D.C., USA \\ ${ }^{5}$ Grupo de Investigación en Física Aplicada, Departamento de Física, Universidad del Norte, Barranquilla, Colombia \\ ${ }^{6}$ Institut für Physik, Johannes Gutenberg Universität Mainz, 55128 Mainz, Germany \\ ${ }^{7}$ Institute of Physics, Czech Academy of Sciences, \\ Cukrovarnická 10, 162 00, Praha 6, Czech Republic
}

(Dated: May 19, 2021)

\begin{abstract}
It is commonly believed that in typical collinear antiferromagnets, with no net magnetization, the energy bands are spin-(Kramers-)degenerate. The opposite case is usually associated with a global time-reversal symmetry breaking (e.g., via ferro(i)magnetism), or with the spin-orbit interaction is combined with the broken spatial inversion symmetry. Recently, another type of spin splitting was demonstrated to emerge in some fully compensated by symmetry, nonrelativistic, collinear magnets, and not even necessarily non-centrosymmetric. These materials feature non-zero spin density staggered not only in real, but also in momentum space. This duality results in a combination of characteristics typical of ferro- and antiferromagnets. Here we discuss this novel concept in application to a well-known semiconductor, $\mathrm{FeSb}_{2}$, and predict that upon certain alloying it becomes magnetic, and features such magnetic duality. The calculated energy bands split antisymmetrically with respect to spin degenerate nodal surfaces (and not nodal points, as in the case of spin-orbit splitting. This combination of a large $(0.2 \mathrm{eV})$ spin splitting, compensated net magnetization and metallic ground-state, and a particular magnetic easy axis generate a large anomalous Hall conductivity $(\sim 150 \mathrm{~S} / \mathrm{cm})$ and a sizable magneto-optical Kerr effect, all deemed to be hallmarks of nonzero net magnetization. We identify a large contribution to the anomalous response originating from the spin-orbit interaction gapped anti-Kramers nodal surfaces, a mechanism distinct from the nodal lines and Weyl points in ferromagnets.
\end{abstract}

PACS numbers:

Antiferromagnets are commonly associated with spindegenerate bands throughout the entire Brillouin zone. The reason is that while antiferromagnets break the timereversal symmetry $\mathcal{T}$ only microscopically, they preserve it, by definition, when combined with another symmetry operation $\mathcal{O}$. Examples of such operations are a lattice translation $\boldsymbol{t}$ (i.e., doubling of the unit cell) or a spatial inversion $\mathcal{P}$. In those cases the combined symmetry and protects the Kramers spin degeneracy for all wavectors.

Recently, it was pointed out that this is not necessarily always the case. There exists magnets where the collinear spin densities (we do not discuss noncollinear spin textures in this paper) are perfectly compensated but their particular spatially anisotropy leads to a strong spin splitting of energy bands[1-6] and surprisingly strong anomalous responses such as spontanous[7] and crystal Hall effect $[1,8]$, crystal magneto-optical Kerr effect[9], spin polarized currents[10,11] and giant magnetoresistance effects $[7,12,13]$. The material base for this emerging class of magnets is potentially large, but so far relatively few materials have been convincingly identified[1, 7, 8] and we only started to explore their fascinating electronic structure. In addition, many of these systems are insulating, eliminating or suppressing many of the effects above, or their ground-state easy axis is incompatible with the anomalous response.

From the above description it follows that such uncon- ventional magnets must involve more than one crystallographically equivalent magnetic site in the nonmagnetic unit cell (otherwise the operation $\mathcal{O}$ would be a lattice translation). Furthermore, if the structure includes at least one bond between two ions with the opposite spins such that its middle point is an inversion symmetry center, this structure is and ordinary antiferromagnet with Kramers degeneracy (such an operation would map a spin-up states onto a spin-down state with the same momentum). Incidentally, the same condition is usually invoked regarding Dzyaloshinskii-Moria interaction.

One way to break this symmetry is to surround magnetic ions by tilted cages of nonmagnetic atoms[1] such as in the marcasite structure. The arguably best-explored marcasite material $\mathrm{FeSb}_{2}$ is experimentally nonmagnetic, albeit exhibits unusual properties including a magnetic response that changes from diamagnetic to paramagnetic. Experimentally, the $\mathrm{FeSb}_{2}$ transport switches from weakly semiconducting at higher temperatures to metallic at $T \sim 50-100 \mathrm{~K}$. The optical gap has been measured between $76 \mathrm{meV}$ [14] and $130 \mathrm{meV}$ [15] and the transport gap is anisotropic with multiple gaps between $4 \mathrm{meV}$ and $36 \mathrm{meV}$ [14-16]. Various density functional theory (DFT) approximations [17-19] have found a pseudogap and semiconducting non-magnetic ground state with an indirect gap. Doping with both $\mathrm{Cr}[21]$ and $\mathrm{Co}[22]$ has been effected, and leads to magnetic phases, some of them 
not entirely determined.

In this paper we predict, using first-principles calculations, that the stoichiometric $\mathrm{FeSb}_{2}$ is, on the mean-field level, an ordinary antiferromagnet with Kramers degenerate bands, with the structure traditionally called AFMe and shown in Fig. 1(a). However, in our calculations this structure is nearly degenerate with another magnetic structure, AFMo (Fig. 1(b)), which is, actually, an unconventional "dual" magnet as descibed above. The calculated energy of the nonmagnetic state is also nearly degenerate with the other two. We argue that the antiferromagnetic state shown in Fig. 1(a), is suppressed in the stoichiometric compound due to to spin fluctuations. Importantly, these energies are close despite the fact that the AFMo structure, being a good metal, is disadvanteged by kinetic energy. Not surprisingly, when this disadvantage is removed by doping $\mathrm{FeSb}_{2}$ away from the semiconducting gap, the unconventional spin-split AFMo state is stabilized, such as for moderate hole $(\mathrm{Cr})$ or electron (Co) doping. Larger dopings stabilize the trivial AFMe structure.

The nonrelativistic band-structure of the AFMo phase exhibits spin-split Fermi surfaces as illustrated in Fig. 1(c), with the nodal planes as shown in Fig. 1(d). The spin splitting follows a higher symmetry than the underlying orthorhombic crystal structure, since the reflection about these plane changes the sign, but not the amplitude of the splitting. It can therefore be expanded into lattice harmonics as

$$
E(\boldsymbol{k}, \uparrow)-E(\boldsymbol{k}, \downarrow)=F(\boldsymbol{k}) \sin \left(k_{x} a\right) \sin \left(k_{y} b\right)
$$

where $F(\boldsymbol{k})$ respects the full underlying lattice symmetry and $a, b$ are the lattice parameters. We refer to the resulting symmetrically spin split bandstructure with Kramers nodal surfaces as anti-Kramers (AK) to distinguish it from the conventional Rashba antisymmetric spin splitting with spin degenerate Kramers points. Furthermore, we show by direct relativistic first-principle calculations that the spin-orbit interaction gapped AK nodal surfaces significantly contribute to a large spontaneous crystal Hall conductivity $\sigma_{x y} \sim 150 \mathrm{~S} / \mathrm{cm}$ and magnetooptical response on the level of $\varepsilon_{x y}(\omega) \sim \pm 10$. We will show that the AK mechanism ensures large Berry curvature at Fermi surfaces. This AK mechanism is very robust, in sharp contrast with the conventional mechanisms operative in ferromagnets, which rely on fine-tuning of nodal lines or Weyl points close to the Fermi level [23-25]. Since $\mathrm{CrSb}_{2}-\mathrm{FeSb}_{2}-\mathrm{CoSb}_{2}$ form a continuous solid solution with a marcasite (pseudo-marcasite for high Co content) structure[21, 22], such doping should be accessible experimentally and thus this system represents a prime candidate for testing the predicted anomalous responses.
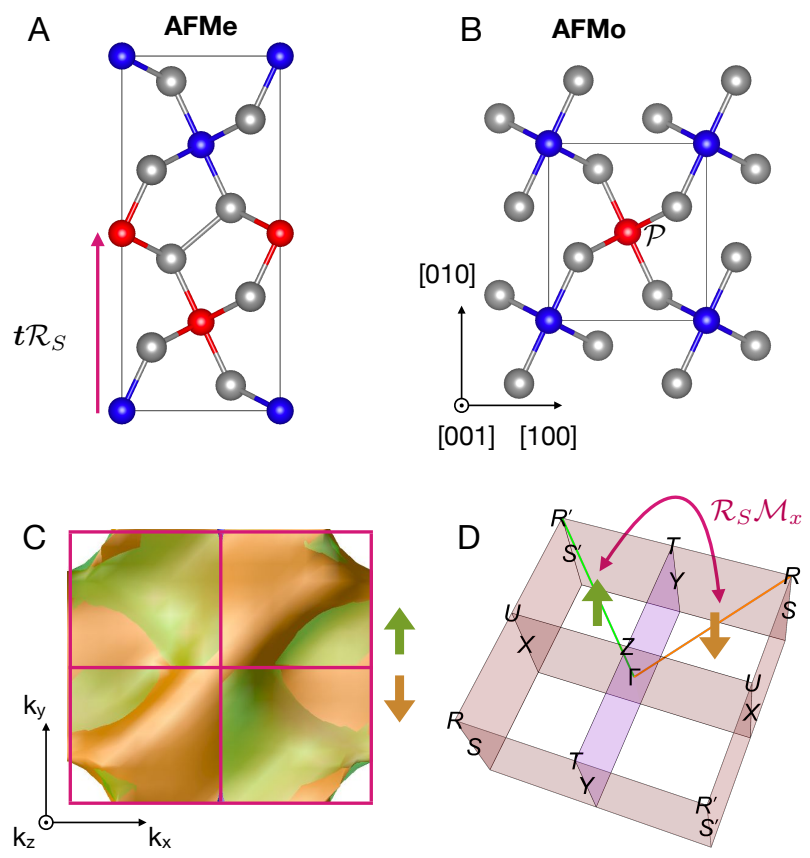

FIG. 1: Magnetic ground-states in $\mathbf{F e S b}_{2}$. The Kramers (a) and spin-split (b) ordering shown on the $\mathrm{FeSb}_{2}$ structure. Fe atoms are shown in red ("up" spin) and blue ("down" spin) and $\mathrm{Sb}$ atoms are shown in light grey. In the $\mathrm{K}$ state we mark the half-unit cell translation coupled with time-reversal $\mathbf{t} \mathcal{T}$. In the AFMo state we mark the inversion symmetry $\mathcal{P}$. (c) Top view along the c-axis on the spin polarized Fermi surface calculated for the spin split antiferromagnetic $\mathrm{FeSb}_{2}$ state. (d) Brillouin zone with marked spin degenerate antiKramers nodal surfaces. We also show the path $\Gamma R$ and $\Gamma R^{\prime}$ to emphasize that it is orbitally degenerate but with an opposite spin polarization (marked by green and orange) and we mark the magenta $\mathcal{R}_{S} \mathcal{M}_{x}$ plane.

\section{ANTIFERROMAGNETIC GROUND-STATES IN FESB $_{2}$}

The marcasite crystal of $\mathrm{FeSb}_{2}$ exhibits the nonmagnetic orthorhombic symmetry space group Pnnm. The previous first principle studies of assumed that the $\mathrm{Fe}$ electrons are strongly correlated, resulting in a competitive meta-ferromagnetic state with Fe magnetization on the order of $1 \mu_{B} \cdot[26]$ However, these calculations did not address any possibility of antiferromagnetic order in $\mathrm{FeSb}_{2}$. Meanwhile, there is study of antiferromagnetism of another marcasite crystal, $\mathrm{CrSb}_{2} \cdot[27]$

In our calculations, we consider two antiferromagnetic orderings shown in Fig. 1(a) and (b). The former, known in the literature as AFMe[27], is an ordinary Kramers antiferromagnet (KAF). It is obtained by quadrupling the nonmagnetic unit cell along [100] and [001] axes. The latter ordering (Fig. 1(b)), known as AFMo[27], preserves the size of the nonmagnetic unit cell and is an AK magnet, where the spin polarization is non-zero, and changes sign both in real (from one Fe to the other), and in reciprocal 
(across the nodal planes) space.

In Fig. 2(a) we show our calculated ground-state energy differences as a function of doping on the Fe site. We employed both virtual crystal approximation (VCA) and supercell first-principle calculations (see Methods). First, we focus on the electron and hole doping around the Fe stoichiometric point corresponding to the blue curve in Fig. 2(a). The KAF is lower in energy than the nonmagnetic state (blue curve) in this region except in the vicinity of the stoichiometric Fe point, where it is only marginally $(\sim 1 \mathrm{meV} / \mathrm{Fe})$ more stable. Enhancing the tendency to magnetism by adding Hubbard U (LDA+U), obviously, stabilizes the KAF magnetic solution even further, albeit, given the experimental situation, $\mathrm{LDA}+\mathrm{U}$ may be in fact less accurate than the straight DFT (see Methods for the choice of the density functional used).

Next, we discuss the energetics of the two antiferromagnetic phases (the green curve in Fig. 2(a)). For the stoichiometric Fe, $\mathrm{Cr}$ and $\mathrm{MnSb}_{2}$ the KAF state is lower than the AK one by, for instance, $\approx 11 \mathrm{meV}$, or $\approx 120$ $\mathrm{K}$ per Fe (see Fig. 2(a)) for pure $\mathrm{FeSb}_{2}$. The lower energy of the KA structure can be related to opening a pseudogap in the density of states, nearly identical to the non-magnetic pseudogap, while the spin split antiferromagnetic phase is a good metal, with the corresponding loss of the one-electron energy. There are two corollaries of this finding: first, the near-degeneracy of two rather different magnetic states, as it is common in frustrated magnetic systems, is liable to suppress both ordered states and stabilize the dynamically nonmagnetic one. Second, one can conjecture that as long as the material is doped away, in either direction, and this disadvantage of the spin split antiferromagnetic phase is alleviated, the latter will become the most stable. These strong fluctuations may be playing an important role in thermoelectricity $\mathrm{FeSb}_{2}$, through an energy dependence of the scattering rate, but such an analysis is beyond the scope of this paper.

As expected, our VCA calculations predicts that moderate electron or hole doping does stabilize the spin split antiferromagnetic order. To illustrate the role of the pseudogap, we compare the $\mathrm{FeSb}_{2}$ density of states with that for the electron-doped $\mathrm{Co}_{0.3} \mathrm{Fe}_{0.7} \mathrm{Sb}_{2}$ in Fig. 2(c) The spin split antiferromagnetic ordering has the highest weight at the Fermi energy in the former, and the lowest in the latter case. Total energy calculations in Fig. 2(a) confirm that in the calculations the AFMo structure is the most stable in same range of either electron and hole doping. The energy gain due to the antiferromagnetism with respect to the nonmagnetic phase grows extremely rapidly with doping, suggesting that except of the close vicinity to the stoichiometric compound the competition is between different magnetic phases.
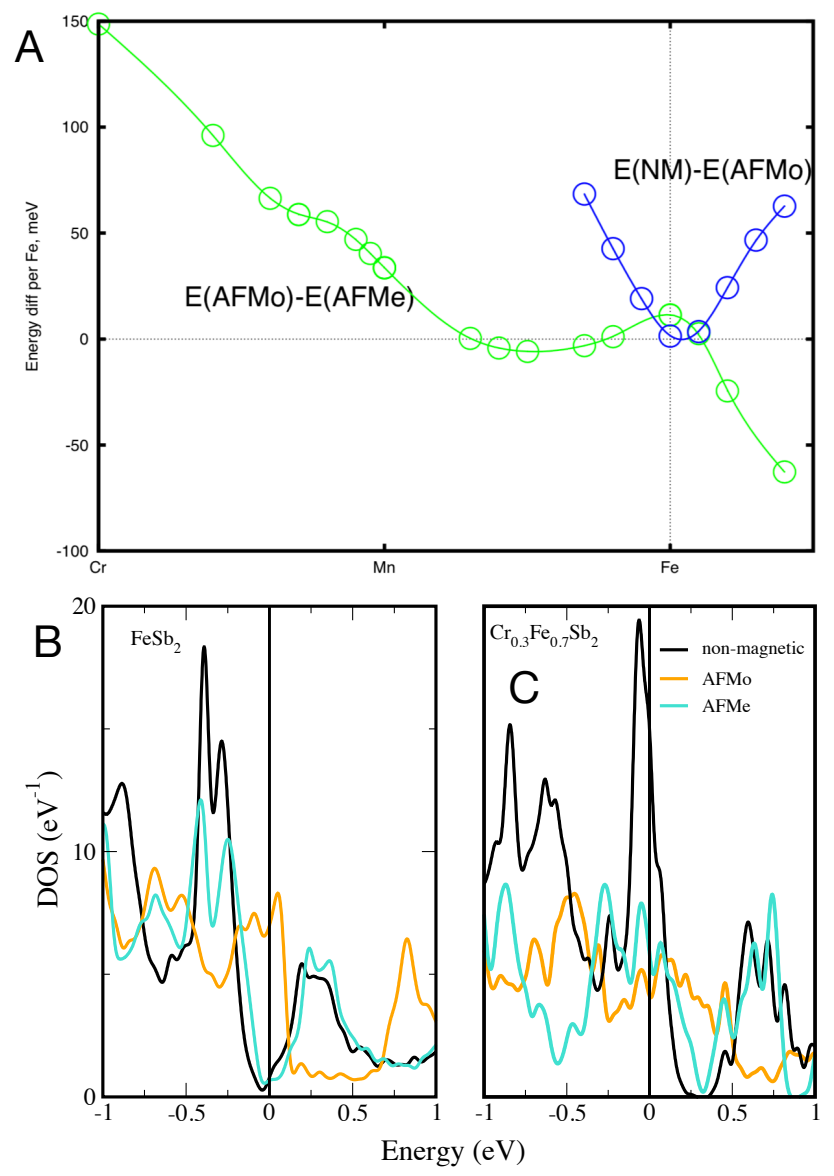

FIG. 2: (a) A plot of the total energy of $\mathrm{FeSb}_{2}$ with different imposed magnetic orderings as a function of hole and electron doping in the virtual crystal approximation (VCA) approximation. The single point at $\mathrm{Cr}=0.5$ corresponds to an actual Cr-doped calculation with full structural relaxation (non-VCA) and illustrates that the VCA captures the relevant energy scale. (b, c) Density of states for $\mathrm{FeSb}_{2}$ and $\mathrm{Co}_{0.3} \mathrm{Fe}_{0.7} \mathrm{Sb}_{2}$ in the VCA, illustrating the removal of weight from $\mathrm{E}_{F}$ upon hole doping in the AFMo and FM patterns, but an increase in weight for non-magnetic state. The KA gains some weight at $\mathrm{E}_{F}$

\section{NONRELATIVISTIC SYMMETRY ANALYSIS OF ANTI-KRAMERS SPIN SPLITTING}

We now consider symmetries of the magnetic phases. The spin degeneracy in the $\mathrm{FeSb}_{2} \mathrm{KAF}$ phase is due to the combined $\boldsymbol{t} \mathcal{T}$ symmetry in the magnetic point group [28]. Alternatively, one can say that it is protected by the combined (as opposed to individual) $\mathcal{P} \mathcal{T}$ symmetry. The antiferromagnetism is constructed by quadrupling the unit cell, i.e., doubling along both the [100] and [001] crystal axes. The opposite magnetic sublattices in the KAF phase are thus connected by the nonmagnetic unit cell translations combined with time-reversal.

Let us now consider the unconventional AK phase. 
There, magnetic order does not change the size of the unit cell and the crystal lacks both $\boldsymbol{t} \mathcal{T}$ and $\mathcal{P} \mathcal{T}$ symmetries, and thus allows for a spin splitting at a general wave vector.

If we neglect the spin-orbit interaction, the real space and the spin space are decoupled. The resulting nonrelativistic symmetry space group[7] of the $\mathrm{FeSb}_{2}$ includes the following symmetry operations:

$$
\left\{\mathcal{E}, \mathcal{P}, \mathcal{M}_{z}, \mathcal{C}_{2 z}\right\}+\boldsymbol{t}^{\prime} \mathcal{R}_{S}\left\{\mathcal{C}_{2 x}, \mathcal{M}_{x}, \mathcal{C}_{2 y}, \mathcal{M}_{y}\right\}
$$

Here the $\mathcal{R}_{S}$ changes the sign of the spin quantization axis, thus being the nonrelativistic analogue of time reversal. The remaining operations $\mathcal{P}, \mathcal{M}$ and $\mathcal{C}_{2}$ are unitary symmetries acting in the real space and these symmetries map each magnetic sublattice onto itself. In contrast, the remaining four symmetries include the $\mathcal{R}_{S}$ and map the two opposite magnetic sublattice on each other, combined with the half unit cell translation $\boldsymbol{t}^{\prime}=\left(\frac{1}{2} \frac{1}{2} \frac{1}{2}\right)$. This reflects the fact that in the nonmagnetic Pnnm group $\mathcal{P}$, $\mathcal{M}_{z}, \mathcal{C}_{2 z}$ are symmorphic operations, while the rest are glide planes and screw axes.

We can understand the anti-Kramers band structure by analyzing the action of the symmetries in the momentum space. Let us now demonstrate the action of $\mathcal{R}_{S} \mathcal{M}_{x}$ :

$$
\mathcal{R}_{S} \mathcal{M}_{x} E\left(k_{x}, k_{y}, k_{z}, \sigma\right)=E\left(-k_{x}, k_{y}, k_{z},-\sigma\right) .
$$

Thus, $E\left(k_{x}, k_{y}, k_{z}, \uparrow\right)=E\left(-k_{x}, k_{y}, k_{z}, \downarrow\right)$, and for $k_{x}=0$ the two spins are degenerate, $E\left(0, k_{y}, k_{z}, \uparrow\right.$ )$=E\left(0, k_{y}, k_{z}, \downarrow\right)$. On the other hand, both spinup and spin-down bands structure must separately respect the Bloch theorem, so $E(\mathbf{k}, \uparrow)=E(\mathbf{k}-\mathbf{G}, \downarrow)$. Using $\mathbf{G}=(2 \pi, 0,0)$, we see that $E\left(-\pi, k_{y}, k_{z}, \uparrow\right)=$ $E\left(\pi, k_{y}, k_{z}, \uparrow\right)=E\left(-\pi, k_{y}, k_{z}, \downarrow\right)$. This set of rules (similarly for $k_{y}$ ) generates the set of the nodal planes shown in Fig. 1, and the functional form of Eq. 1. Note that while the location of such nodal surfaces depends on the exact nature of the operations in the second term in Eq. (2), their presence is a universal feature of AK magnets ( $c f$., e.g., previously discussed tetragonal $\mathrm{RuO}_{2}[1], \mathrm{MnO}_{2}[5]$ and $\left.\mathrm{MnF}_{2}[4]\right)$.

Away from the nodal planes the absolute value of the spin splitting obeys the full nonmagnetic crystal symmetry, while it sign alternates[7], which ensures that the material as a whole is spin-compensated by symmetry. To illustrate this, we plot in Fig.3 bands along the path $\Gamma R R^{\prime} \Gamma$ shown in Fig. 1(d). The full spin splitting and nodal structure can be seen in the Fermiology throughout the BZ shown in Fig. 1(d) and in SI for all dopings. In SI we list the degeneracies along the high symmetry lines and planes in the Brillouin zone in Tables S1 and S2.

We emphasize the distinction of the spin splitting by antiferromagnetism and by relativistic interactions. The spin-orbit spin splitting (e.g., Rashba or Ising in 2D) is characterized by spin-degenerate points at the timereversal symmetry invariant momenta protected by the time-reversal symmetry. Away from these points, the bands spin-split antisymmetrically,

$$
E(\boldsymbol{k}, \uparrow)=E(-\boldsymbol{k}, \downarrow),
$$

i.e., the spin splitting $E(\boldsymbol{k}, \uparrow)=E(-\boldsymbol{k}, \downarrow)$ follows a $p-$, or $f$-wave rather than $d$-wave, as in Eq. 1, symmetry. This, of course, is related to the fact that spin orbit splitting can only happen in noncentrosymmetric crystals, while in the absence of spin-orbit the $E(\boldsymbol{k}, \sigma)=E(-\boldsymbol{k}, \boldsymbol{\sigma})$ holds independent of the magnetic structure (the time reversal symmetry can be applied to the Schrödinger equation for each spin separately).

\section{RELATIVISTIC SYMMETRY ANALYSIS AND ELECTRONIC STRUCTURE}

From the experimental point of view the most important prediction is that the easy magnetization axis for $\mathrm{FeSb}_{2}$ is calculated to be [010] (see Table I). Indeed, as mentioned in the introduction, while AK magnetism is,

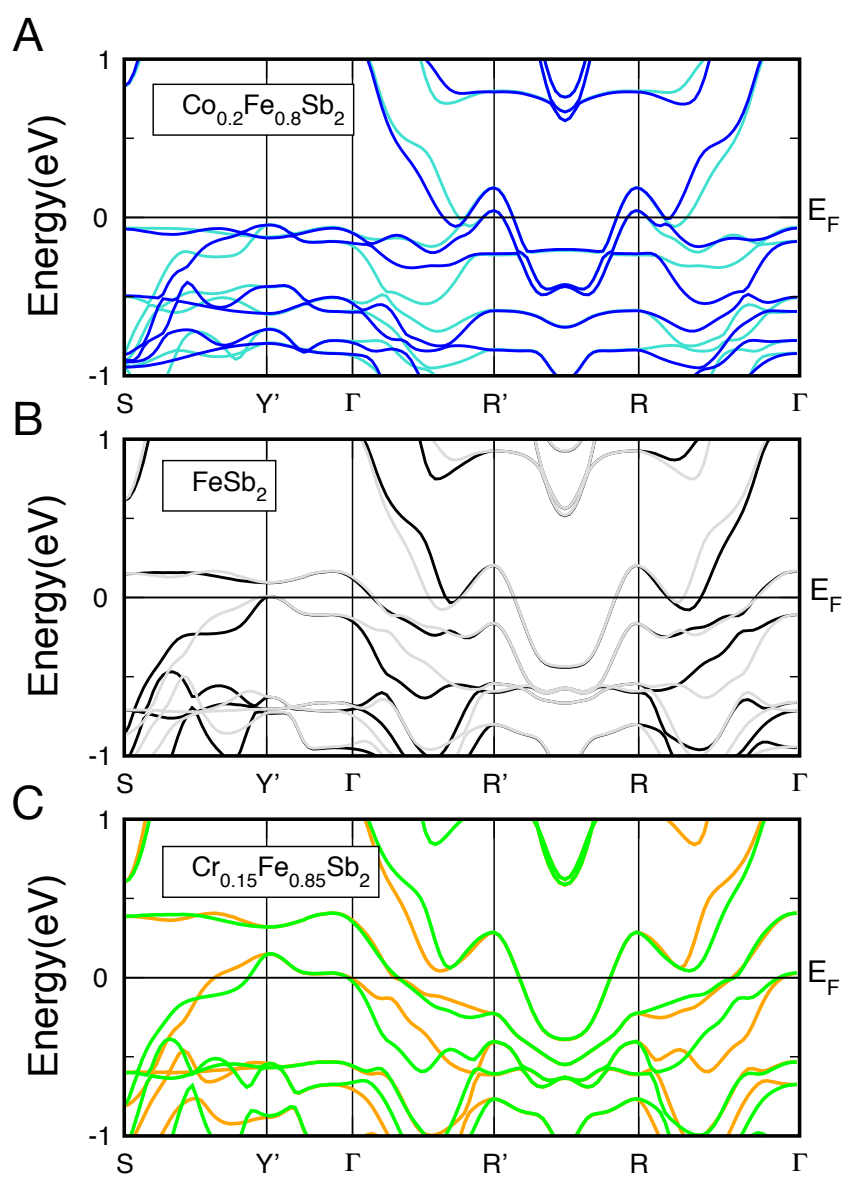

FIG. 3: The nonrelativistic band structure of $\mathrm{FeSb}_{2}$ with the AK pattern, for electron doped (top), stoichiometric (middle) and hole doped (bottom) and corresponding Fermi surfaces. The "up" and "dn" bands split along low symmetric momenta in accordance with Table S1 and S2. Furthermore, the splitting exactly reverses along perpendicular momenta, i.e. $\Gamma R$ and $\Gamma R^{\prime}$. This explains the overall net zero moment. 


\begin{tabular}{llll} 
& $\mathrm{FeSb}_{2}$ & $\mathrm{Cr}_{0.15} \mathrm{Fe}_{0.85} \mathrm{Sb}_{2}$ & $\mathrm{Co}_{0.2} \mathrm{Fe}_{0.8} \mathrm{Sb}_{2}$ \\
\hline \hline$\sigma_{y z}$ & 143 & -31 & 94 \\
\hline next favorable axis $[001]$ & {$[001]$} & {$[010]$} \\
\hline easy axis & {$[010]$} & {$[010]$} & {$[001]$} \\
\hline MAE & -0.12 & -0.25 & -0.12 \\
\hline
\end{tabular}

TABLE I: The Hall conductivity in $\mathrm{S} / \mathrm{cm}$ and the easy axis with magnetic anisotropy energy in $\mathrm{meV}$ relative to the next favorable axis ([001]).

in principle, not that rare, those so far few identified as metals do not necessary have the magnetization direction conducive for anomalous transport (as discussed, for instance, w.r.t. $\mathrm{RuO}_{2}$ in Ref. [1]). In $\mathrm{FeSb}_{2}$, to be specific, two out of three orthorhombic directions manifest the latter ([100] and [010]), and the third does not (Table I; see also Tables S1 and S2 in the SI for the spin-orbit induced splittings). Fortunately, Cr (but not Co) doping not only preserves the favorable magnetic anisotropy, but even enhances it. In Fig. 4(a), we show the calculated magnetization density isosurfaces for the magnetization along the [010] direction (calculated in VASP, see Methods). The anisotropic spin densities highlight the breaking of the $\boldsymbol{t} \mathcal{T}$ and $\mathcal{P} \mathcal{T}$ symmetries.

In the following we will illustrate how the symmetry analysis of the spin splitting proceeds in the relativistic case. In this case we need to take into account the full magnetic space group (MSG), which depends on the selected spin quantization axis; for instance, a mirror plane $\mathcal{M}_{y}$ conserves the spin components $s_{y}$, but flips the directions of $s_{x}$ and $s_{z}$. For the spin direction along the easy axis [010] direction the MSG is $P n n^{\prime} m^{\prime}$ (i.e., the glide and the mirror planes symmetries for $y$ and $z$ planes, respectively, are preserved only when combined with the time-reversal symmetry) and includes the following symmetry operations $\left(\boldsymbol{t}^{\prime}=\left[\frac{1}{2} \frac{1}{2} \frac{1}{2}\right]\right)$ :

$$
\{\mathcal{E}, \mathcal{P}\}+\boldsymbol{t}^{\prime}\left\{\mathcal{C}_{2 x}, \mathcal{M}_{x}\right\}+\mathcal{T}\left\{\mathcal{C}_{2 z}, \mathcal{M}_{z}\right\}+\boldsymbol{t}^{\prime} \mathcal{T}\left\{\mathcal{C}_{2 y}, \mathcal{M}_{y}\right\}
$$

The notations are the same as in Eq. (2), but the spatial and spin space symmetries are now coupled, resulting in a different structure of the symmetry group and band degeneracies. We also note that the $P n n^{\prime} m^{\prime}$ MSG describes a type-III antiferromagnet, that is, preserving the nonmagnetic unit cell.

In Fig. 4(b) we show the calculated relativistic energy bands with spins along [010] direction and including spinorbit. By comparing these bands with the nonrelativistic ones we confirm the collinear antiferromagnetism as the main source of the spin-splitting in $\mathrm{FeSb}_{2}$. The spinorbit interaction additionally splits certain high symmetry planes, lines and points as summarized in Tables S1, 2. However, the relativistic spin splitting is much weaker.

Not all nodal planes shown in Fig. 1(d) are preserved, but one plane, $k_{y}= \pm \pi$, is. Let us explain why. Let us denote $\left\{\boldsymbol{t}^{\prime} \mid \mathcal{T} \mathcal{C}_{2 y}\right\}=\mathcal{T} \mathcal{S}_{2 y}$. The space operation alone, $\mathcal{S}_{2 y}$, is translating the first $\mathrm{Fe}, \mathrm{Fe} 1$, into its AF counterpart, $\mathrm{Fe} 2$. If the spin of $\mathrm{Fe} 1(2)$ is $s_{y}\left(-s_{y}\right), \mathcal{S}_{2 y}$ alone is not a symmetry operation, but $\mathcal{T} \mathcal{S}_{2 y}$. Conversely, for the spin $s_{x}\left(-s_{x}\right), \mathcal{S}_{2 y}$ is a symmetry operation, but not $\mathcal{T} \mathcal{S}_{2 y}$. The same is true for $s_{z}$. This is why for the spins along $x, y$ or $z$, the MSG is, respectively, $P n^{\prime} n m^{\prime}, P n n^{\prime} m^{\prime}$ and $P n n m$.

We will now demonstrate the symmetry protection of nodal surfaces. Let us now consider two Bloch functions, $\varphi_{\mathbf{k}}$ and $\varphi_{\mathbf{k}}^{\prime}$ related by the nonsymmorphic symmetry operation $\mathcal{O}=\mathcal{T} \mathcal{S}_{2 y}$ :

$$
\begin{aligned}
E_{\mathbf{k}^{\prime}} \varphi_{\mathbf{k}^{\prime}}^{\prime}(\mathbf{r}) & =H(\mathbf{r}) \varphi_{\mathbf{k}^{\prime}}^{\prime}(\mathbf{r})=H(\mathbf{r})\left[\mathcal{O} \varphi_{\mathbf{k}}(\mathbf{r})\right] \\
\mathcal{O}\left[H(\mathbf{r}) \varphi_{\mathbf{k}}(\mathbf{r})\right] & =E_{\mathbf{k}}\left[\mathcal{O} \varphi_{\mathbf{k}}(\mathbf{r})\right]
\end{aligned}
$$

Here we should remember that $\mathbf{k}^{\prime}=\mathcal{O} \mathbf{k} \neq \mathbf{k}$, and that $\mathcal{O}$ is a symmetry operation of the Hamiltonian, $\mathcal{O} H(\mathbf{r}) \mathcal{O}^{-1}=H(\mathbf{r})$. We see that the energies $E_{\mathbf{k}}$ and $E_{\mathbf{k}^{\prime}}$ of the two Bloch states, $\varphi_{\mathbf{k}}(\mathbf{r})$ and $\varphi_{\mathbf{k}^{\prime}}^{\prime}(\mathbf{r})$, are identical. That means that either $\varphi_{\mathbf{k}}(\mathbf{r})$ and $\varphi_{\mathbf{k}^{\prime}}^{\prime}(\mathbf{r})$ are identical, or these are two genuinly degenerate states. In order to demonstrate the latter, we calculate the overlap of the two functions:

$$
\left\langle\varphi_{\mathbf{k}^{\prime}}^{\prime}(\mathbf{r}) \mid \varphi_{\mathbf{k}}(\mathbf{r})\right\rangle=\left\langle\mathcal{O} \varphi_{\mathbf{k}^{\prime}}^{\prime}(\mathbf{r}) \mid \mathcal{O} \varphi_{\mathbf{k}}(\mathbf{r})\right\rangle=\mathcal{O}^{2}\left\langle\varphi_{\mathbf{k}}(\mathbf{r}) \mid \varphi_{\mathbf{k}}^{\prime}(\mathbf{r})\right\rangle
$$

Now, by definition, $\mathcal{O}^{2}=\boldsymbol{t}^{\prime} \mathcal{T} \mathcal{C}_{2 y} \boldsymbol{t}^{\prime} \mathcal{T} \mathcal{C}_{2 y}=$ $e^{i\left(-k_{x}+k_{y}-k_{z}\right) / 2} e^{i\left(k_{x}+k_{y}+k_{z}\right) / 2}=e^{i k_{y}}$ so $\left\langle\varphi_{\mathbf{k}}(\mathbf{r}) \mid \varphi_{\mathbf{k}^{\prime}}^{\prime}(\mathbf{r})\right\rangle=$ $e^{i k_{y}}\left\langle\varphi_{\mathbf{k}}(\mathbf{r}) \mid \varphi_{\mathbf{k}^{\prime}}^{\prime}(\mathbf{r})\right\rangle$, that is to say, $\varphi_{\mathbf{k}}(\mathbf{r})$ and $\varphi_{\mathbf{k}^{\prime}}^{\prime}(\mathbf{r})$ are not identical, moreover, orthogonal, unless $k_{y}=0$. Next, we note that two vectors, $k_{y}=\pi$ and $k_{y}^{\prime}=-\pi$, are related by the operation $\mathcal{O}=\mathcal{T} \mathcal{S}_{2 y}$, and at the same time by the reciprocal lattice vector $G=2 \pi$. This proves that at the plane $k_{y}=\pi$ there are two orthogonal wave functions giving the same electron energy, even with the spin-orbit, if the sublattice magnetization direction is $y$. Obviously, the same proof applies to $k_{x}=\pi$ and the sublattice magnetization direction $x$.

We note that our protecting antiunitary screw symmetry is oriented along the antiferromagnetic sublattices and its orientation can be controlled by the magnetic quantization axis (along $x$ or $y$ direction). This contrasts the ferromagnetic nodal surfaces protected by antiunitary screw axis present for magnetization perpendicular to the symmetry axis[29].

In Fig. 4(d) we show the spin polarization at the Fermi surface. We observe that most of the Fermi sheets are spin-polarized, but near the former nodal planes $k_{x, y}=0$ the spin direction continuously rotates from one direction to the opposite. This feature makes the $\mathrm{FeSb}_{2}$ antiferromagnet a promising spin current generator [10].

Note that for the spins along [001] crystal axis, when the MSG is Pnnm, the three mirror symmetries prohibit the existence of a ferromagnetic pseudovector and thus spontaneous Hall conductivity [1]. For spins along [100] the MSG allows a non-zero anomalous Hall conductivity and magnetooptical (nondiagonal) components of the dialectric function, which are $\sigma_{x z}\left(\sigma_{y z}\right)$ for $P n^{\prime} n m^{\prime}$ $\left(P n n^{\prime} m^{\prime}\right)$. 

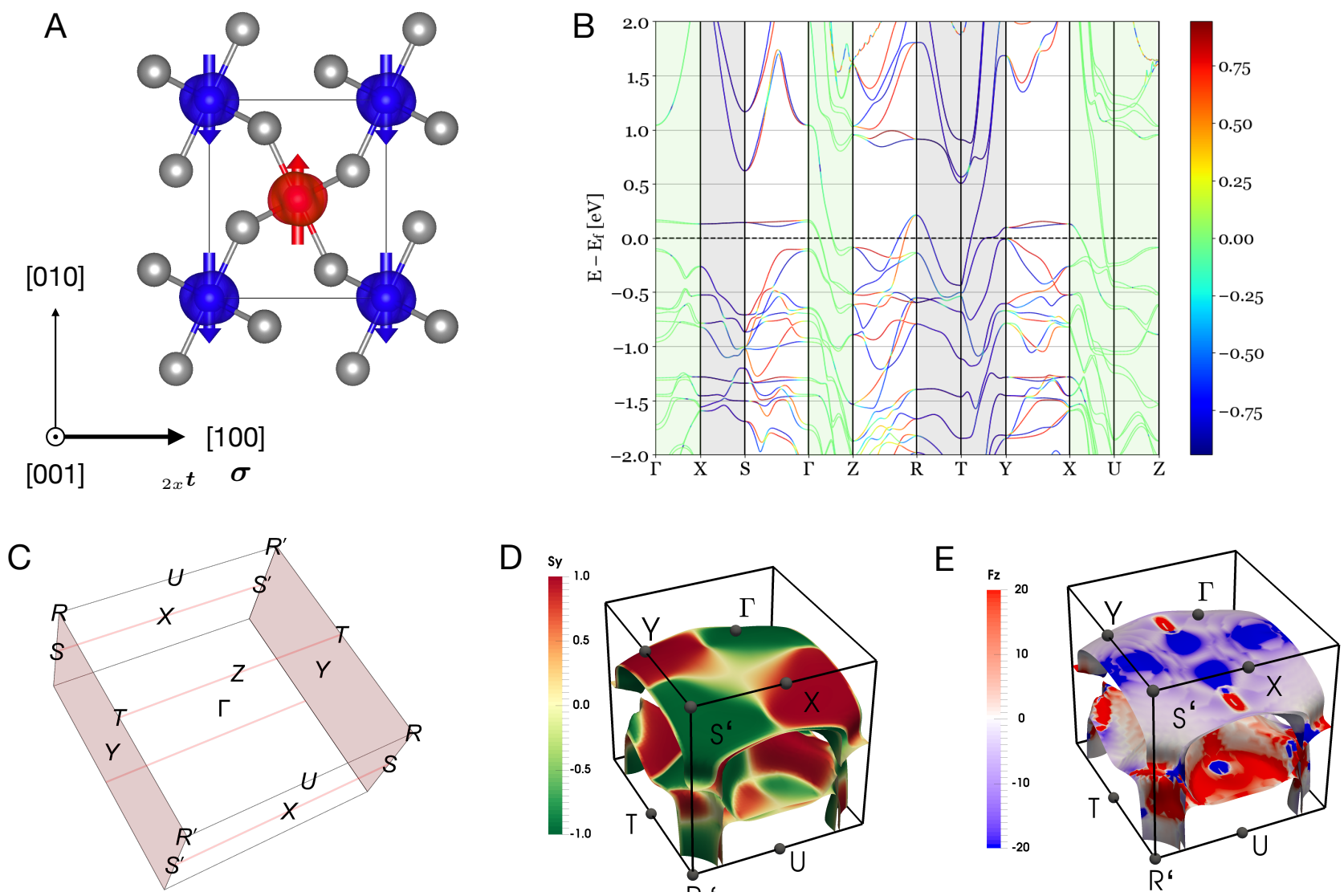

FIG. 4: Electronic structure of FeSb 2 with spin-orbit interaction. (a) Unit cell with the calculated magnetization densities and marked symmetry group generators $\mathcal{P}, \mathcal{C}_{2 a} t$, and $\mathcal{C}_{2 z} \mathcal{T}$, and Hall vector $\boldsymbol{\sigma}$. (b) Spin projected energy bands calculated with spin-orbit interaction and sublattice magnetization along [010] crystal direction. The grey shaded panels are spin degenerate, and green shaded ones are spin-split by spin-orbit, but still not spin polarized. (c) Brillouin zone band degeneracy manifolds in the presence of spin-orbit interaction are marked by the red shading. (d) Spin projected Fermi surfaces along [010] quantization axis. (e) Berry curvature resolved on Fermi surface reveals large contributions from gapped AK nodal features at $k_{x, y}=0$ planes marked by high-intensity blue and red colour.

\section{ANOMALOUS ELECTRIC AND MAGNETOOPTICAL RESPONSE}

Berry curvature. There are non-trivial ramifications in the anomalous response of the momentum spacedependent splitting and presence of AK nodal surfaces. We start by discussing the Berry curvature calculations shown in Fig. 4(e) and Fig. S2. We observe that the large Berry curvature originates from the regions of the Brillouin zone which are degenerate without spin-orbit interaction, The presence of the spin-orbit interaction splits these nodal features and generates anisotropic Berry curvature as we show in Fig. 4(e), including the former nodal planes. The fact that the AKAF nodal manifolds are surfaces, and not lines, ensures a large Berry curvature contribution from regions in the Brillouin zone where the nodal surface intersects the mirror planes.

Crystal Hall effect. The Hall vector direction follows simple rules[1]. The Hall vector $\boldsymbol{\sigma}$ is parallel to the rotational axis of $\mathcal{C}_{2 x} \boldsymbol{t}^{\prime}$ symmetry as shown in Fig. 4(a). The amplitude of the intrinsic spontaneous Hall effect can be calculated by integrating the Berry curvature over the Brillouin zone. We see in Fig. 5(a) that while the Berry curvature changes sign, the cancellation is incomplete the Hall conductivity $\sigma_{y z}$ is nonzero, while $\sigma_{x y}$ and $\sigma_{x z}$ vanish, in agreement with our symmetry analysis [1]. We show the resulting energy dependence of the Hall conductivity in Fig. 5(a) for the three doping levels discussed in Section II (see also Fig. S3).

At the Fermi level, we obtain, for the [010] spin direction, $143 \mathrm{~S} / \mathrm{cm}$ for the undoped compound and $94 \mathrm{~S} / \mathrm{cm}$ and $-31 \mathrm{~S} / \mathrm{cm}$ for the Co- and Cr-doped compounds, respectively. Note however that the Co-doped compound has calculated [001] easy axis, which does no afford an anomalous response. We conclude that the Cr-doped $\mathrm{FeSb}_{2}$ is the best candidate for the experimental observation of the spontaneous crystal Hall effect. Previously, the crystal Hall effect was experimentally observed in $\mathrm{RuO}_{2}$, but only after a spin-reorientation transition, induced by 

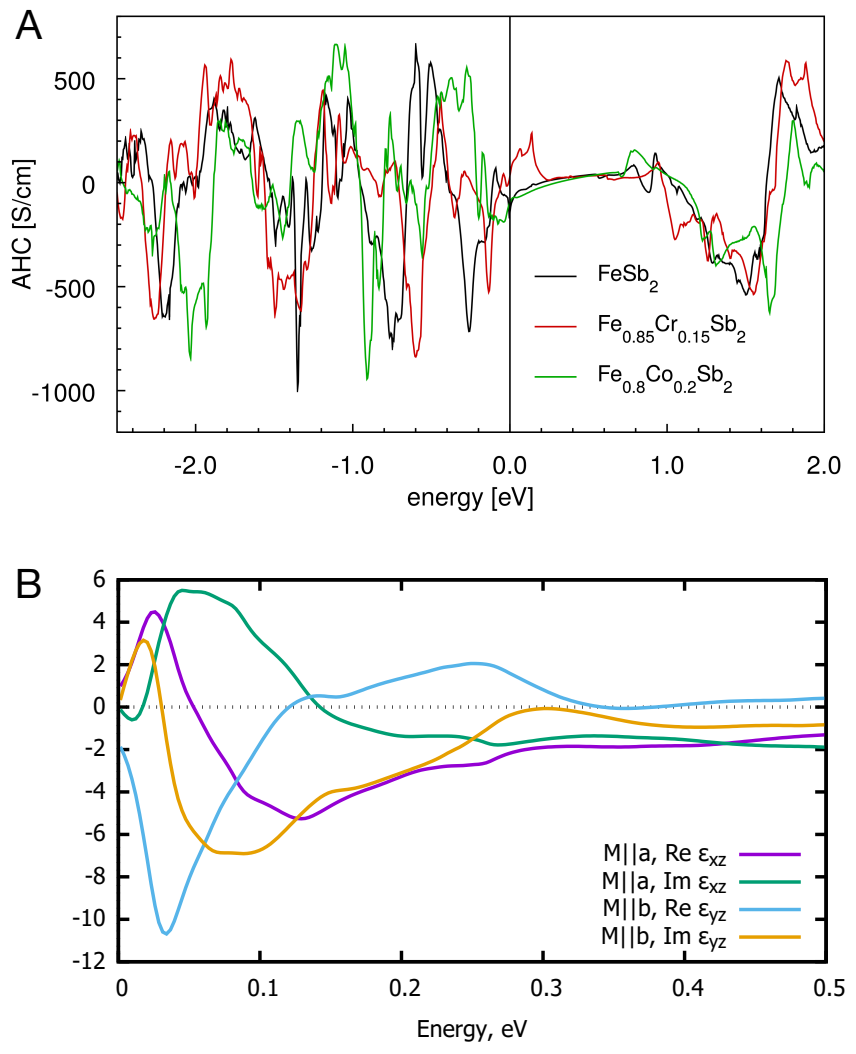

FIG. 5: Anomalous charge and optical response. (a) Energy dependence of the crystal Hall conductivity on the energy for the stoichiometric $\mathrm{FeSb}_{2}$, and electron and hole doped variants. (b) Energy (frequency) dependence of the off-diagonal optical conductivity tensor calculated in stoichiometric $\mathrm{FeSb}_{2}$, which determines the magnetooptical Kerr effect[9].

a strong external magnetic field [8].

Crystal magnetooptical Kerr effect. The magnetooptical Kerr effect (MOKE) is a dynamic counterpart of the direct-current anomalous Hall effect and follows the analogous symmetry-determined selection rules. It is a method of choice for checking for ferro- and ferrimagnetic transition and is routinely used as a litmus test for nonzero net magnetization and/or noncollinear antiferromagnets[30-33]. In accordance to that, the easy axis that affords a sizable Hall conductivity also suggests, contrary to the common wisdom, presence of magnetooptical effects in this fully compensated collinear material.

The complex Kerr rotation angle is proportional, in this case, to the $y z$ component of the complex dielectric function, $\varepsilon_{y z}(\omega)$. The proportionality coefficient depends on the diagonal (Drude) dielectric function, and thus on the sample-dependent relaxation rate. For that reason, we present in Fig. 5(b) only the corresponding nondiagonal components of the interband dielectric function, $\operatorname{Re} \varepsilon_{i j}(\omega)$ and $\operatorname{Im} \varepsilon_{i j}(\omega)$ calculated from first-principles (see Methods). No that the $\omega \rightarrow 0$ limit of the intraband con- ductivity tensor, $\sigma_{i j}(\omega)=\frac{\omega}{4 \pi} \operatorname{Im} \varepsilon_{i j}(\omega)$, is the anomalous Hall conductivity[30]. In agreement with the symmetry analysis and the Hall effect calculations, we find for the spin along [010] $([100])$ a nonzero $\varepsilon_{y z}(\omega)\left(\varepsilon_{x z}(\omega)\right)$. We obtain the largest magnitude for the $\sigma_{y z}(\omega)$ at $\omega \approx 0.8 \mathrm{eV}$, which is $\sim 5$ times larger than the dc Hall conductivity. The magnitude of the magnetetooptical effects in $\mathrm{FeSb}_{2}$ is comparable to that in typical ferromagnets.

Methods We used the Vienna Ab-initio Simulation Package (VASP) [34] with the PBE-GGA approximation to the exchange correlation potential [35] and PAW pseudopotentials [36] to fully relax $\mathrm{FeSb}_{2}$ in a variety of magnetic patterns. We found that not only was the AFMe pattern the lowest in energy, but the resulting lattice parameters of $a=5.8379, b=6.5248$, and $c=3.1811$ matched extremely well with the measured parameters of $a=5.8328, b=6.53758$, and $c=3.19730$ [37].

To verify the energy orderings of the different magnetic orderings with the highest possible accuracy, we calculated each one with the FPLO code [38], also with the PBE-GGA approximation, but adding spin-orbit coupling via a fully relativistic four component solution to the Dirac equation. We found that the energy differences shifted by less than $0.2 \mathrm{meV}$ due to the inclusion of this term. To simulate doping, we used the Wien2k code [39] (again with PBEGGA) and employed the Virtual Crystal Approximation (VCA) with the structure held constant according to the measurements of Ref. [37]. This shifts the charge of the ion cores to an average of the two charged species being simulated. This method allows electrons/holes to be added to the system in an average, band-like manner, consistent with experimental measurements that find a lack of local moments in favor of a fluctuating itinerant magnetic state $[40,41]$. To check the validity of the VCA, we simulated $\mathrm{Fe}_{0.5} \mathrm{Cr}_{0.5} \mathrm{Sb}_{2}$ with real, rather than virtual, $\mathrm{Cr}$ doping and calculated the magnetic energies of the various magnetic patterns (see Fig. 2(a)).

In our VASP calculations of the bandstructure and magnetisation densities in Figure 4 we set the energy cutoff to $520 \mathrm{eV}$, use the momentum mesh of $7 \times 6 \times 12$, and we use the GGA potential with the Vosko-Wilk-Nusair potential. Furthermore, we use the Wannier90 code[42] to construct the Wannier functions. We calculate the spontaneous Hall conductivity by integrating the Berry curvature in Brillouin zone in the WannierTools code [43]. To corroborate the results for the Hall conductivity and to extend them to the doped cases $\mathrm{Cr}_{0.15} \mathrm{Fe}_{0.85} \mathrm{Sb}_{2}$ and $\mathrm{Co}_{0.2} \mathrm{Fe}_{0.8} \mathrm{Sb}_{2}$ via $\mathrm{VCA}$ we repeated the calculation using maximally projected Wannier functions (WFs)[44] as provided by FPLO (version 19.00) in full relativistic mode with subsequent integration of the Berry curvature. We projected onto a set of $\mathrm{Fe} 3 d$ and $\mathrm{Sb} 5 p$ orbitals, which generates WFs for all bands in $[-6,6] \mathrm{eV}$ with only tiny Wannier fit errors of about $3 \mathrm{meV}$. The Berry curvature was calculated by the method of Ref. [45] using only the dominant term (which was checked to be a rather small error). The integration of the Berry curvature using the Wannier model was performed with a k-mesh 
of $301 \times 301 \times 602$ and $400 \times 400 \times 800$ points for the undoped and doped compounds, respectively, which was checked to be converged to a below 5 percent error for the Hall conductivity at the Fermi level. Calculations of the optical response were performed with the Wien2k code [39].

Acknowledgements LS acknowledges the EU FET
Open RIA Grant No. 766566 and SPIN+X Grant (DFG SFB TRR 173). LS and RGH acknowledge the computing time granted on the supercomputer Mogon at Johannes Gutenberg University Mainz (hpc.uni-mainz.de). IM acknowledges support from the U.S. Department of Energy through Grant No. DE-SC0021089. MJ was supported by ONR through the NRL basic research program.
[1] L. Šmejkal, R. Gonzalez-Hernandez, T. Jungwirth, and J. Sinova, Science Advances 6, eaaz8809 (2020).

[2] K.-H. Ahn, A. Hariki, K.-W. Lee, and J. Kuneš, Physical Review B 99, 184432 (2019), ISSN 2469-9950, URL https : //link.aps.org/doi/10.1103/PhysRevB.99.184432.

[3] S. Hayami, Y. Yanagi, and H. Kusunose, J. Phys. Soc. Japan 88, 123702 (2019).

[4] L.-D. Yuan, Z. Wang, J.-W. Luo, E. Rashba, and A. Zunger, Phys. Rev. B 102, 014422 (2020).

[5] Y. Noda, K. Ohno, and S. Nakamura, Physical Chemistry Chemical Physics 18, 13294 (2016), ISSN 1463-9076, URL http://xlink.rsc.org/?DOI=C5CP07806G.

[6] S. López-Moreno, A. H. Romero, J. Mejía-López, A. Muñoz, and I. V. Roshchin, Phys. Rev. B 85, 134110 (2012), URL https://link.aps.org/doi/10. 1103/PhysRevB.85.134110.

[7] H. Reichlova, R. Lopes Seeger, R. González-Hernández, I. Kounta, R. Schlitz, D. Kriegner, P. Ritzinger, M. Lammel, M. Leiviskä, V. Petřiček, et al., Tech. Rep. (2020), 2012.15651v1, URL https://arxiv.org/pdf/ 2012.15651.pdf.

[8] Z. Feng, X. Zhou, L. Šmejkal, L. Wu, Z. Zhu, H. Guo, R. González-Hernández, X. Wang, H. Yan, P. Qin, et al. (2020), 2002.08712, URL http://arxiv.org/abs/2002. 08712 .

[9] K. Samanta, M. Ležaić, M. Merte, F. Freimuth, S. Blügel, and Y. Mokrousov, Journal of Applied Physics 127, 213904 (2020), ISSN 0021-8979, URL https://doi.org/10.1063/5.0005017http://aip. scitation.org/doi/10.1063/5.0005017.

[10] R. González-Hernández, L. Šmejkal, K. Výborný, Y. Yahagi, J. Sinova, T. Jungwirth, and J. Železný, Physical Review Letters 126, 127701 (2021), ISSN 0031-9007, 2002.07073, URL http://arxiv.org/abs/2002.07073https://link. aps.org/doi/10.1103/PhysRevLett.126.127701.

[11] M. Naka, Y. Motome, and H. Seo (2020), 2011.12459, URL https://arxiv.org/pdf/2011.12459.pdfhttp:// arxiv.org/abs/2011.12459.

[12] L. Šmejkal, A. B. Hellenes, R. González-Hernández, J. Sinova, and T. Jungwirth (2021), 2103.12664, URL http://arxiv.org/abs/2103.12664.

[13] D.-F. Shao, S.-H. Zhang, M. Li, and E. Y. Tsymbal (2021), 2103.09219, URL http://arxiv.org/abs/2103.09219.

[14] C. Homes, Q. Du, C. Petrovic, W. Brito, S. Choi, and G. Kotliar, Sci. Rep. 8, 11692 (2018).

[15] A. Herzog, M. Marutzky, J. Sichelschmidt, F. Steglich, S. Kimura, S. Johnsen, and B. Iversen, Phys. Rev. B 82, 245205 (2010).

[16] P. Sun, N. Oeschler, S. Johnsen, B. B. Iversen, and F. Steglich, 39, 1012 (2010).

[17] C.-J. Kang and G. Kotliar, Phys. Rev. Mat. 2, 034604
(2018).

[18] J. Tomczak, K. Haule, T. Miyake, A. Georges, and G. Kotliar, Phys. Rev. B 82, 085104 (2010).

[19] A. Bentien, G. Madsen, S. Johnsen, and B. Iversen, Phys. Rev. B 74, 25105 (2006).

[20] S. Sebastian, private communication (2020).

[21] R. Hu, V. Mitrović, and C. Petrovic, Phys. Rev. B 76, 115105 (2007).

[22] R. Hu, V. Mitrović, and C. Petrovic, Phys. Rev. B 74, 195130 (2006).

[23] D. Gosálbez-Martínez, I. Souza, and D. Vanderbilt, Physical Review B 92, 085138 (2015), ISSN 1550235X, 1505.07727, URL https://link.aps.org/doi/10.1103/ PhysRevB.92.085138.

[24] K. Kim, J. Seo, E. Lee, K.-T. T. Ko, B. S. Kim, B. G. Jang, J. M. Ok, J. Lee, Y. J. Jo, W. Kang, et al., Nature Materials 17, 1 (2018), ISSN 14764660, URL http://www . nature.com/articles/s41563-018-0132-3.

[25] E. Liu, Y. Sun, N. Kumar, L. Muechler, A. Sun, L. Jiao, S.-Y. Y. Yang, D. Liu, A. Liang, Q. Xu, et al., Nature Physics 14, 1125 (2018), ISSN 1745-2473, URL http: //www . nature.com/articles/s41567-018-0234-5http: //dx.doi.org/10.1038/s41567-018-0234-5.

[26] A. V. Lukoyanov, V. V. Mazurenko, V. I. Anisimov, M. Sigrist, and T. M. Rice, The European Physical Journal B - Condensed Matter and Complex Systems 53 (2006).

[27] G. Kuhn, S. Mankovsky, H. Ebert, M. Regus, and W. Bensch, Phys. Rev. B 87, 085113 (2013).

[28] L. Šmejkal, J. Železný, J. Sinova, and T. Jungwirth, Physical Review Letters 118, 106402 (2017), ISSN 0031-9007, 1610.08107, URL https://link.aps.org/doi/10.1103/ PhysRevLett.118.106402.

[29] W. Wu, Y. Liu, S. Li, C. Zhong, Z. M. Yu, X. L. Sheng, Y. X. Zhao, and S. A. Yang, Physical Review B 97, 115125 (2018), ISSN 24699969, 1712.09773.

[30] W. Feng, G. Y. Guo, J. Zhou, Y. Yao, and Q. Niu, Physical Review B 92, 144426 (2015), ISSN 1550235X, 1509.02865 .

[31] T. Higo, H. Man, D. B. Gopman, L. Wu, T. Koretsune, O. M. J. van 't Erve, Y. P. Kabanov, D. Rees, Y. Li, M.-T. Suzuki, et al., Nature Photonics 12, 73 (2018), ISSN 1749-4885, 1805.06758, URL http://www.nature. com/articles/s41566-017-0086-z.

[32] W. Feng, J.-P. Hanke, X. Zhou, G.-Y. Guo, S. Blügel, Y. Mokrousov, and Y. Yao, Nature Communications 11, 118 (2020), ISSN 2041-1723, URL http://www.nature. com/articles/s41467-019-13968-8.

[33] N. Sivadas, S. Okamoto, and D. Xiao, Physical Review Letters 117, 267203 (2016), ISSN 0031-9007, URL https://link.aps.org/doi/10.1103/PhysRevLett. 117.267203https://journals.aps.org/prl/pdf/10. 
1103/PhysRevLett.117.267203.

[34] G. Kresse and J. Hafner, Phys. Rev. B 47, 558 (1993).

[35] J. P. Perdew, K. Burke, and M. Ernzerhof, Phys. Rev. Lett. 77, 3865 (1996).

[36] P. E. Blöchl, Phys. Rev. B 50, 17953 (1994).

[37] C. Petrovic, J. Kim, S. Bud'ko, A. Goldman, and P. Canfield, Phys. Rev. B 67, 155205 (2003).

[38] K. Koepernik and H. Eschrig, Phys. Rev. B 59, 1743 (1999), URL https://link.aps.org/doi/10.1103/ PhysRevB.59.1743.

[39] P. Blaha, K. Schwarz, G. K. H. Madsen, D. Kvasnicka, and J. Luitz, Wien2k (2002), iSBN 3-9501031-1-2.

[40] T. Koyama, H. Nakamura, T. Kohara, and Y. Takahashi, J. Phys. Soc. Japan 79, 093704 (2010).

[41] I. A. Zaliznyak, A. T. Savici, V. O. Garlea, R. Hu, and
C. Petrovic, Phys. Rev. B 83, 184414 (2011).

[42] G. Pizzi, V. Vitale, R. Arita, S. Blügel, F. Freimuth, G. Géranton, M. Gibertini, D. Gresch, C. Johnson, T. Koretsune, et al., Journal of Physics: Condensed Matter 32, 165902 (2020), URL https://doi.org/10.1088\% $2 \mathrm{~F} 1361-648 \mathrm{x} \% 2 \mathrm{Fab} 51 \mathrm{ff}$.

[43] Q. S. Wu, S. N. Zhang, H. F. Song, M. Troyer, and A. A. Soluyanov, Computer Physics Communications 224, 405 (2017), ISSN 00104655, 1703.07789.

[44] H. Eschrig and K. Koepernik, Phys. Rev. B 80, 104503 (2009), URL https://link.aps.org/doi/10. 1103/PhysRevB.80.104503.

[45] X. Wang, J. R. Yates, I. Souza, and D. Vanderbilt, Phys. Rev. B 74, 195118 (2006). 


\title{
Supplementary information:
}

\section{Anti-Kramers antiferromagnetism in doped $\mathrm{FeSb}_{2}$}

\author{
I. I. Mazin,${ }^{1,2}$ K. Koepernik, ${ }^{3}$ M.D. Johannes, ${ }^{4}$ \\ Rafael González-Hernández, ${ }^{5,6}$ and Libor Šmejkal ${ }^{6,7}$ \\ ${ }^{1}$ Department of Physics and Astronomy, \\ George Mason University, Fairfax, USA \\ ${ }^{2}$ Center for Quantum Science and Engineering, \\ George Mason University, Fairfax, USA \\ ${ }^{3}$ Institute for Theoretical Solid State Physics, \\ IFW Dresden, 01069 Dresden, Germany \\ ${ }^{4}$ Center for Computational Materials Science, \\ Naval Research Laboratory, Washington D.C., USA \\ ${ }^{5}$ Grupo de Investigación en Física Aplicada, Departamento de Física, \\ Universidad del Norte, Barranquilla, Colombia \\ ${ }^{6}$ Institut für Physik, Johannes Gutenberg Universität Mainz, 55128 Mainz, Germany \\ ${ }^{7}$ Institute of Physics, Czech Academy of Sciences, \\ Cukrovarnická 10, 162 00, Praha 6, Czech Republic
}

(Dated: May 19, 2021)

ГX XS SY YГ ГZ XU SR YT ZU UR RT TZ

\begin{tabular}{lllllllllllll}
\hline \hline no SOC & $\checkmark$ & $\checkmark$ & $\checkmark$ & $\checkmark$ & $\checkmark$ & $\checkmark$ & $\checkmark$ & $\checkmark$ & $\checkmark$ & $\checkmark$ & $\checkmark$ & $\checkmark$ \\
\hline 100 & & $\checkmark$ & $\checkmark$ & & & $\checkmark$ & $\checkmark$ & & $\checkmark$ & $\checkmark$ & & \\
\hline 010 & & $\checkmark$ & $\checkmark$ & & & & $\checkmark$ & $\checkmark$ & & & $\checkmark$ & $\checkmark$ \\
\hline 001 & $\checkmark$ & $\checkmark$ & $\checkmark$ & $\checkmark$ & $\checkmark$ & & $\checkmark$ & & & & & \\
\hline arbitrary & & $\checkmark$ & $\checkmark$ & & & & & & & & \\
\hline
\end{tabular}

TABLE I. Spin degeneracy of high symmetry lines in the Brillouin zone without (line 2) and with (lines 3-6) spin-orbit interaction. The first column indicates the spin quantization axis for relativistic calculations. 
A
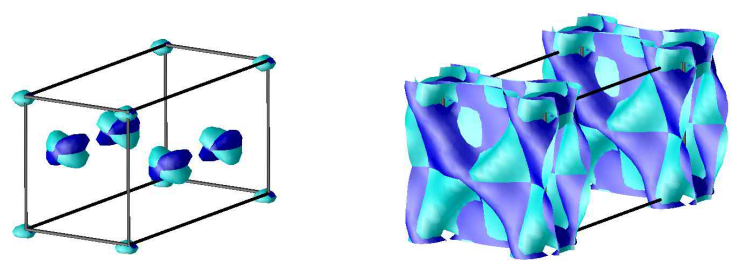

B
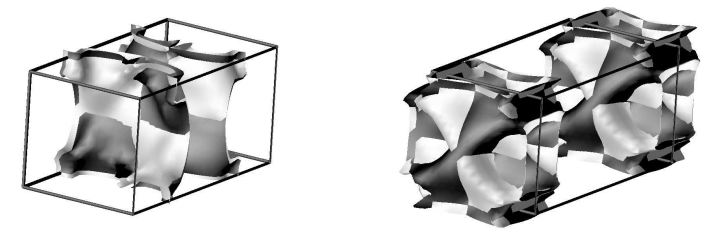

C
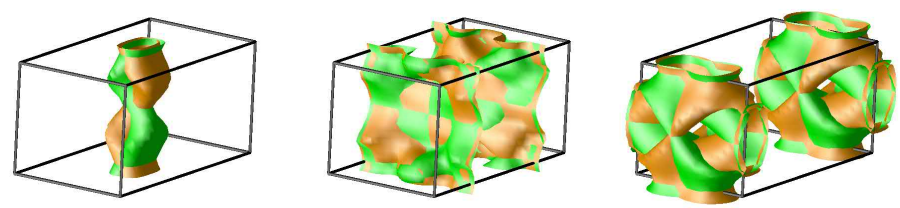

Figure S 1. Calculated Fermi surface pockets for: (a) $\mathrm{Co}_{0.2} \mathrm{Fe}_{0.8} \mathrm{Sb}_{2}$, (b) $\mathrm{FeSb}_{2}$, and (c) $\mathrm{Cr}_{0.15} \mathrm{Fe}_{0.85} \mathrm{Sb}_{2}$. The orientation of the Brillouin zone is shown in panel (d). Note that number of pockets increases with the number of electrons.

ГXUZ ГYTZ XSRU SYTR ZURT ZURT

\begin{tabular}{lllll}
\hline \hline no SOC & $\checkmark$ & $\checkmark$ & $\checkmark$ & $\checkmark$ \\
\hline 100 & & $\checkmark$ & \\
\hline 010 & & $\checkmark$ \\
\hline 001 & & &
\end{tabular}

TABLE II. Same as in Tab. I, for high symmetry planes . 

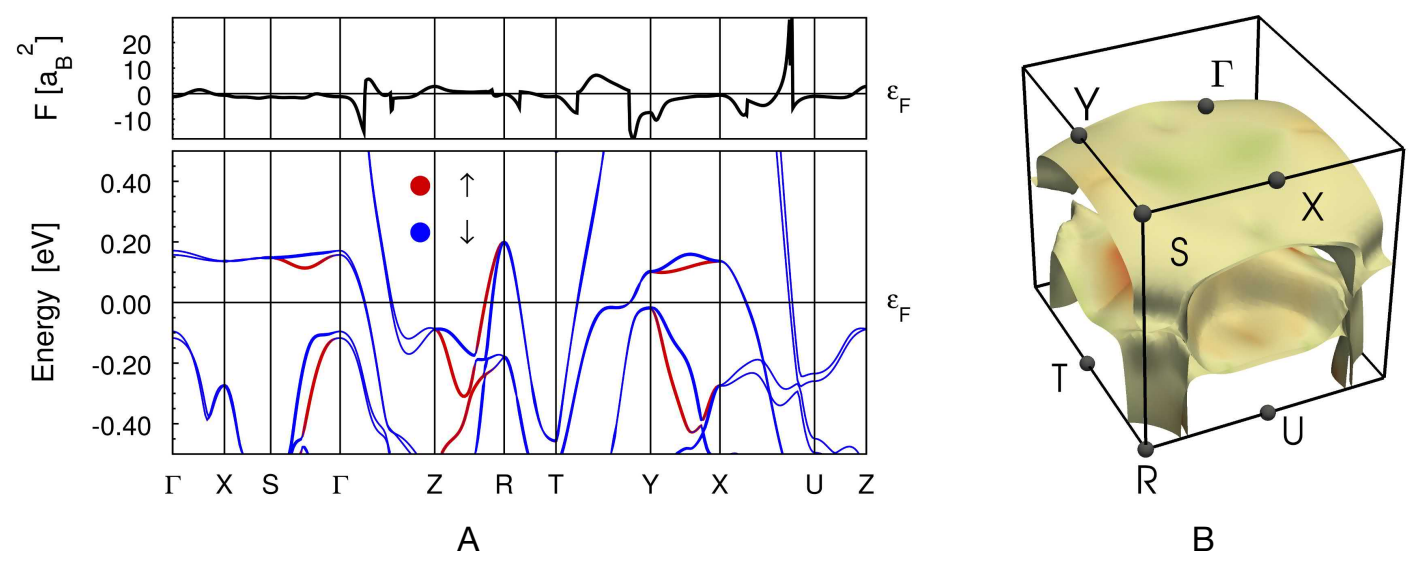

Figure S 2. (a) Calculated energy bands and Berry curvature $\mathcal{F}$ along high symmetry path in momentum space. (b) Spin polarization $S_{y}$ along the [010] direction plot on top of the Fermi surface demonstrates vanishing net moment along the momenta with a large Berry curvature, cf. Fig. 4(e) in main text. The plots were calculated in the FPLO code, see Methods.?????

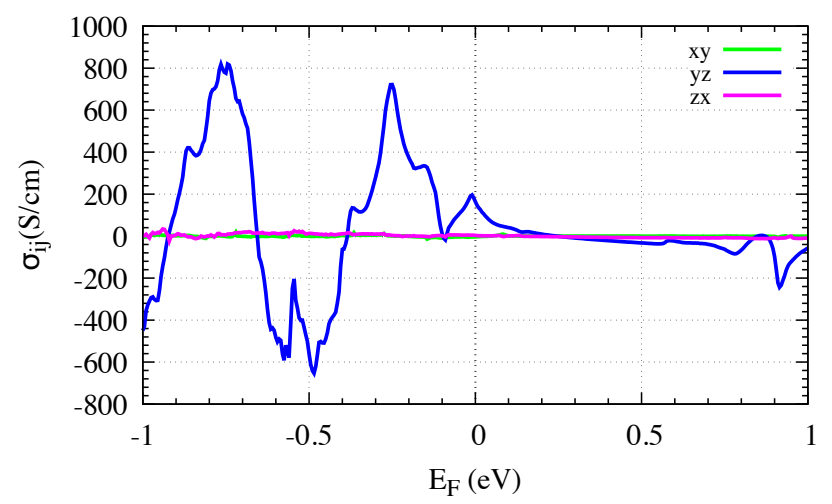

Figure S 3. Calculated spontanous intrinsic crystal Hall conductivity as a function of the Fermi energy, calculated using VASP and Wannier90 codes, for the spin direction [010]. Note that, in agreement with the symmetry analysis in the main text, the only nonzero component is $\sigma_{y z}$. 